\title{
Finger ECG De-noising Based on GA-wavelet Shrinkage
}

\author{
Shenshen Zou ${ }^{1}$ and Xiaohong Zhang ${ }^{2, *}$ \\ ${ }^{1}$ School of Communication Engineering, Hangzhou Dianzi University, Hangzhou Zhejiang 310018, China \\ ${ }^{2}$ School of Electronic Information Engineering, Hangzhou Dianzi University, Hangzhou Zhejiang 310018, China \\ *Corresponding author
}

\begin{abstract}
In view of the low signal to noise ration and the difficulty on de-noising for ECG signal collected under unconstrained environment, a GA(Generation Algorithm) based wavelet method of finger ECG de-noising is proposed after a systematic study of wavelet threshold de-noising and generation algorithm. This new algorithm can dynamically get the optimal solution to fit the threshold in accordance with the characteristic of finger ECG. Then the algorithm is verified by fitting ECG and finger ECG. Compared with other algorithm, results show that the proposed method get the better performance according to the performance index including the output SNR, correlation coefficient and root mean square.
\end{abstract}

Keywords-finger ECG; generation algorithm; wavelet de-noising

\section{INTRODUCTION}

ECG(Electrocardiogram) is a comprehensive reflection of the heart of the continuous contraction and relaxation activities that contains relevant information for human identification. Since ECG is the endogenous signal and featured active, identification using ECG as raw material has more advantages that other biometric do not have. Currently, the research on ECG identification are limited when users under constraint environment, including sensors, ECG acquisition position and mode. Accompanying with the emergence of ECG chips with the features of small size, lower power and easy integration recently, finger based ECG identification can be realized that acquisition of the ECG signal from fingers with high accuracy under non-constrained environment. Compared to traditional clinical ECG acquisition mode, finger based ECG identification which suitable for portable mobile scene has broader prospects. However it also faces enormous challenge, the challenge come from the instability of information acquisition process. ECG is a time-varying signal, so there are more factors can affect the change of ECG waveform when ECG acquisition under non restraint environment. Research reveal that ECG signal acquired by non-clinical standard position has low signal to noise ration(SNR) and more susceptible to kinds of noise, like EMG(electromyography), baseline drift, $50 \mathrm{~Hz}$ and others. In this paper, a new finger ECG wavelet de-noising algorithm based on generation algorithm is proposed, which can dynamically get the optimal solution to fit the threshold in accordance with the characteristic of finger ECG. The proposed can make a least reconstruction error between the reconstructed signal and the original signal and achieve a better de-noising.

\section{METHOD}

Due to the ECG signal contain more noise and has low signal to noise ratio(SNR) with signal collected by finger, the de-noised ECG is not ideal after being wavelet de-noising with the VisuShrink threshold. In this paper, a new finger ECG de-noising method based on wavelet transform and generation algorithm is proposed with the threshold obtained by generation algorithm which changed in accordance with the characteristic of finger ECG. The flow chart of proposed method is shown in Figure 1. First, the suitable wavelet function and wavelet decomposition scale are determined according to the finger ECG character. Then, obtain the optimal value $\beta$ by fitness function and using $\beta$ to fix the threshold $\lambda$, as in (1). Last, after achieving the correction of wavelet coefficient with the pre-obtained threshold, using reconstruction to get clean ECG signal.

$$
\lambda_{j}=\beta \sigma_{j} \sqrt{2 \ln N}
$$

where $\sigma_{j}$ is the noise variance in layer $\mathrm{j}, \mathrm{N}$ is the length of signal.

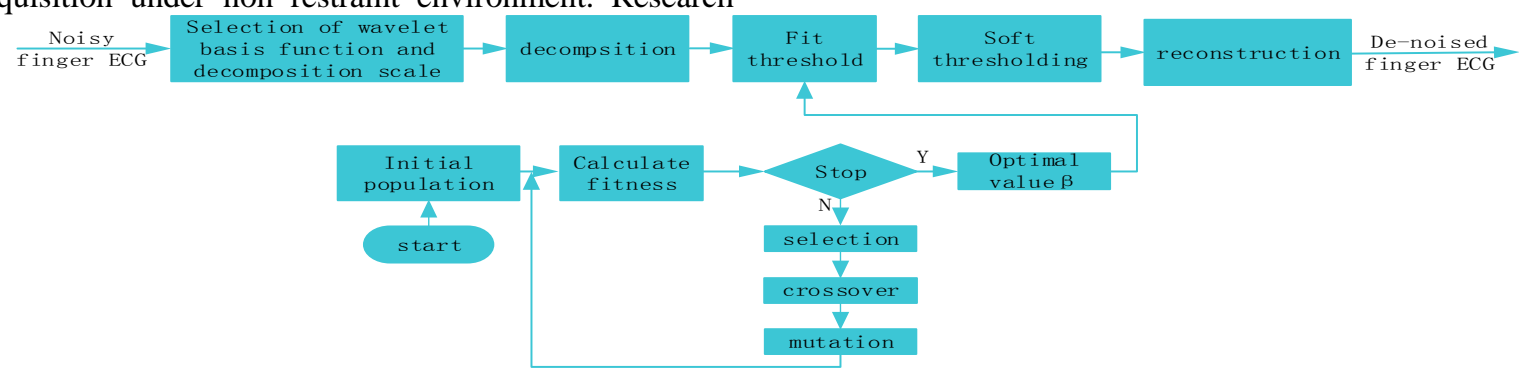

FIGURE I. THE FLOW CHART OF THE NEW FINGER ECG DE-NOISING BASED ON WAVELET TRANSFORM AND GENERATION ALGORITHM 


\section{A. Wavelet Threshold Denoising Algorithm}

In the process of acquisition and transmission, the signal is inevitably introduced into noise, and the presence of noise makes it difficult to process and analyze the signal. Donoho et al. proposed a wavelet threshold de-noising algorithm[1]. First, the signal is decomposed into different scales using the frequency characteristic of the noise and useful signal; Then, shrinkage is played to eliminate noise according to the threshold of wavelet coefficients; Last, reconstruction is implied on the shrinking wavelet coefficients to get the clean signal. The specific steps are follow:

- $\quad$ Select the wavelet decomposition scale and threshold function, imply decomposition on signal $f(x)$ and obtain the wavelet coefficients $W_{k, j}$;

- $\quad$ Threshold on wavelet coefficients $W_{k, j}$ to get $\hat{W}_{k, j}$, and make sure the distance $\left\|\hat{W}_{k, j}-W_{k, j}\right\|$ is small;

- Reconstruction on wavelet coefficients $\hat{W}_{k, j}$ to get clean signal $\hat{f}(x)$.

There are several ways to determine the threshold in wavelet threshold de-noising, one of the most common threshold is VisuShrink threshold [2], as in

$$
\lambda_{j}=\sigma_{j} \sqrt{2 \ln N}
$$

where $\sigma_{i}$ is the standard variance and $\mathrm{N}$ is the length of signal.

Due to the variance of the noise is difficult to know in advance in (2), Donoho et al. [3] proposed an estimation using the intermediate value of wavelet coefficients for variance, as in

$$
\hat{\sigma}_{j}=\frac{\text { median }\left(\left|W_{k, j}\right|\right)}{0.6745}
$$

Tewfic et al. determined a new threshold value by using a constant c to modify VisuShrink threshold, as in

$$
\lambda_{j}=c \sigma_{j} \sqrt{2 \ln N}
$$

Studies have shown that the effect of de-noising use (4) as threshold is better than VisuShrink threshold. And results reveal de-noising using the threshold in (4) can retain image details when c is between 0.2-0.3. However this threshold still lacks universality and applicability, as de-noising with (4) threshold is not ideal to some signals like ECG.

The signal is processed by the threshold function after the threshold value is determined. And two kinds of threshold function are commonly used, which are hard threshold function and soft threshold function.

Hard threshold function:

$$
\hat{W}_{k, j}=\left\{\begin{array}{l}
W_{k, j},\left|W_{k, j}\right| \geq \lambda_{j} \\
0,\left|W_{k, j}\right|<\lambda_{j}
\end{array}\right.
$$

where $W_{k, j}$ is the wavelet coefficient, $\lambda_{j}$ is the threshold value corresponding to the $\mathrm{j}$ layer wavelet coefficient; The wavelet coefficient is modified by 0 if $\left|W_{k, j}\right|$ is less than $\lambda_{j}$, otherwise maintaining the wavelet coefficient unchanged.

Soft threshold function:

$$
\hat{W}_{k, j}=\left\{\begin{array}{l}
\operatorname{sign}\left(W_{k, j}\right)\left(\operatorname{abs}\left(W_{k, j}\right)-\lambda_{j}\right),\left|W_{k, j}\right| \geq \lambda_{j} \\
0,\left|W_{k, j}\right|<\lambda_{j}
\end{array}\right.
$$

where $\operatorname{sign}(\cdot)$ is the symbolic function; The wavelet coefficient is modified by $0 \mathrm{if}\left|W_{k, j}\right|$ is less than $\lambda_{j}$, otherwise the wavelet coefficient is processed by threshold value.

\section{B. Genetic Algorithm}

Genetic algorithm(GA) is proposed by professor Holland which based on the idea of biological evolution and genetics[4] In this algorithm, regarding the problem to be solved as chromosomes, the key is to find the optimal solution of the problem with the basic criterion of "survival of the fittest, not those out”. Studies shown it is a convergent global search algorithm.

The genetic algorithm is realized through three processes, including selection, crossover and mutation. First, a new generation of chromosomes is generated by crossover and mutation. Then, according to the size of fitness, the best individual as the group member of next generation is selected among the previous and next generations. Last, through continuous iteration, the best chromosome is selected as the optimal solution or local optimal solution.

Its specific implementation steps include follows.

- Parameters of genetic algorithm settings: Before starting the genetic algorithm, the parameters of the algorithm should be set up, including population size, chromosome length, selection operation mode, iteration number, crossover probability, mutation probability and so on. The normal circumstances size of the population is $10-200$, crossover probability is generally between $0.25-0.75$, mutation probability is generally between 0.01-0.2.

- Encoding and decoding: Encoding on the needed optimal value should be carried out first when solving a problem. The method of encoding contains real encoding and binary encoding.

- Initial population: According to the size of a given population and the length of the chromosome, the initial population is generated randomly or according to the given requirements ;

- Selection operation: According to fitness distribution, 
this operation is to select the superior individual to the next generation population, where the fitness function as a measuring function for solving the optimal solution. Roulette and elite alternatives are the common ways to realize selection.

- Crossover operation: The crossover operator includes single point crossover, two point crossover and multi-point crossover. The difference between these crossover operators is the number of crossing points on the chromosome.

- Mutation operation: The purpose is to make the genetic algorithm has a certain local search ability while maintaining the diversity of the population and accelerate the algorithm converges to the optimal solution.

- Iteration stops: The iteration stop when the iteration reaching a given value, or the optimal value tends to be stable and so on.

\section{The Source of Finger ECG and Parameter Setting of The Proposed Method}

In this paper, a least reconstruction error will be get between the reconstructed signal and the original signal and achieve a better de-noising with the proposed method, where the threshold is fitted by an optimal value by genetic algorithm.

Herein the experimental data of finger based ECG come from Check Your Biosignal Here initiative(CYBHi) database[5], Surface ECG data[6] and Finger ECG data that collected by ourselves. The sampling of each database is $1000 \mathrm{~Hz}$.

In the choice of wavelet basis function, because of the Singh's study found that the de-noising effect of db8 wavelet in ECG signal denoising is the best[7], so this paper choose db8 as the basis of wavelet. And also select 8 layers of wavelet decomposition for finger ECG as the sampling rate is $1000 \mathrm{~Hz}$. determined by the amount of computation and the convergence speed after several simulations.

As know that the smaller of $\left\|\hat{W}_{k, j}-W_{k, j}\right\|$, the less of reconstruction error between reconstructed signal and original signal. The optimal value $\beta$ obtained when the root mean square error(RMSE) reaches the minimum, which is equivalent to the maximum value of the -RMSE. So choose the -RMSE as fitness function in genetic algorithm.

The selection of select operation is elite mode as it's easy to loss excellent individuals for roulette operation. In the crossover operation, select single-point crossover way and the crossover probability is 0.6 . In mutation operation, also select a single point mutation and the mutation probability is 0.01 .

In (1), due to the unknown source of noise(that is, the unknown of variance $\sigma_{j}$ ), choose the intermediate value of wavelet coefficients (median $\left(\left|W_{k, j}\right|\right) / 0.6745$ )as the estimated noise variance, where $\mathrm{N}$ is the length of signal.

Taking into account the existence of Pesudo-Gibbs effect[8], select soft threshold function for de-noising.

\section{EXPERIMENT AND RESULTS}

To verify the performance of the proposed method, first, produce a clean synthetic ECG signal and plus with varying degrees of noise. Then the de-noising experiments are based on three methods, including wavelet soft thresholding, de-noising based on translation invariant wavelet transform and overlapping group shrinkage[9], the proposed method. Noise cancellation performance is measured by the SNR, RMSE and correlation coefficient. Last, randomly select three signals from the three finger ECG database pointed previous and de-noised by the proposed method in this paper.

In Figure 2 is a clean synthetic ECG signal with length of 10000 points, and the sampling rate is $1000 \mathrm{~Hz}$.

In genetic algorithm, choose decimal encoding to reduce the amount of storage space. And the population size is 10 as

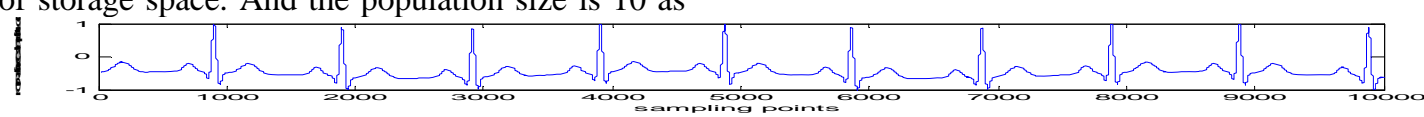

FIGURE II. THE CLEAN SYNTHETIC ECG SIGNAL

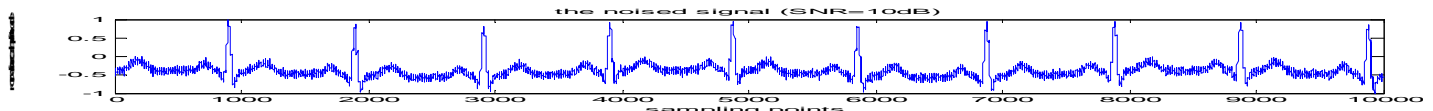

FIGURE III. THE NOISED ECG WITH SNR OF 10DB

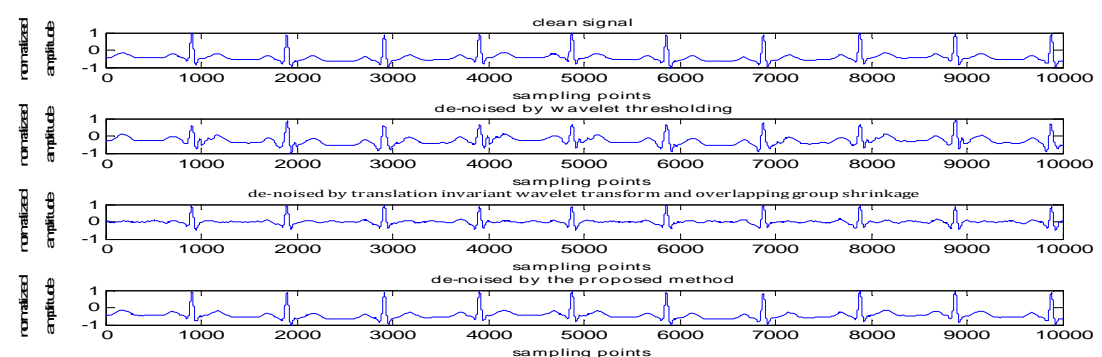

FIGURE IV. THE DE-NOISED RESULTS OF THREE METHOD WITH SNR OF 10DB 


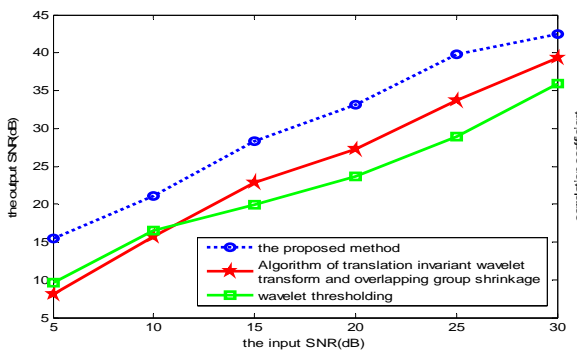

FIGURE V. THE CHANGE OF OUTPUT SNR OF THREE DE-NOISE METHOD WITH DIFFERENT INPUT SNR

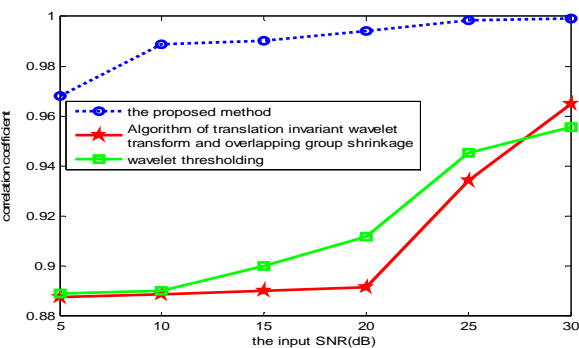

FIGURE VI. THE CHANGE OF CORRELATION COEFFICIENT OF THREE DE-NOISE METHOD WITH

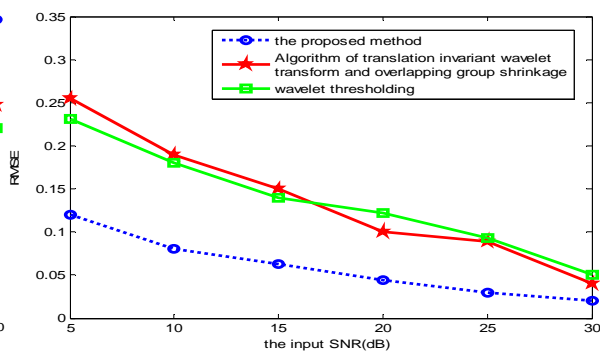

FIGURE VII. THE CHANGE OF RMSE OF THREE DE-NOISE METHOD WITH DIFFERENT INPUT SNR DIFFERENT INPUT SNR

The clean synthetic ECG signal is plus with varying degree noise, and the input SNR start from $5 \mathrm{~dB}$ to $30 \mathrm{~dB}$ with interval of 5dB. The de-noised signal with different SNR are experimented by de-noise methods including wavelet soft thresholding, de-noising based on translation invariant wavelet transform and overlapping group shrinkage, and the proposed method. Figure 3 shows a noisy synthetic ECG with SNR of $10 \mathrm{~dB}$. Figure 4 shows the experimental results of the signal with SNR of $10 \mathrm{~dB}$ that de-noised by three methods respectively. As can be seen from Fig. 4, an obvious sharp wave near the $\mathrm{S}$ complex and some distortion exist in $\mathrm{R}$ complex when signal de-noised with wavelet de-noising; The $\mathrm{Q}$ and $\mathrm{T}$ complexes also have obviously distortion by using de-noising based on translation invariant wavelet transform and overlapping group shrinkage; There is a significant similarity between the original clean signal and the de-noised signal that de-noised by proposed method. Figure 5-figure 7 are respectively represents the changes of output SNR, correlation coefficient and RMSE of three de-noise methods with different input SNR. As can be seen from Figures 5-7, the output SNR and correlation coefficient are the maximum while the RMSE is minimum of the proposed method under different input SNR compared to other two methods, so the proposed method advanced in de-noising than the other two methods.

From the above results, the method of wavelet de-noising algorithm based on generation algorithm is superior than wavelet de-noising and de-noising based on translation invariant wavelet transform and overlapping group shrinkage, than experiments are carried out by three finger signals that randomly selected from the three finger database using the proposed de-noising method. The comparison between before and after of de-noising signal are shown in Figure 8 -10. And noise in the three signals are basically removed.

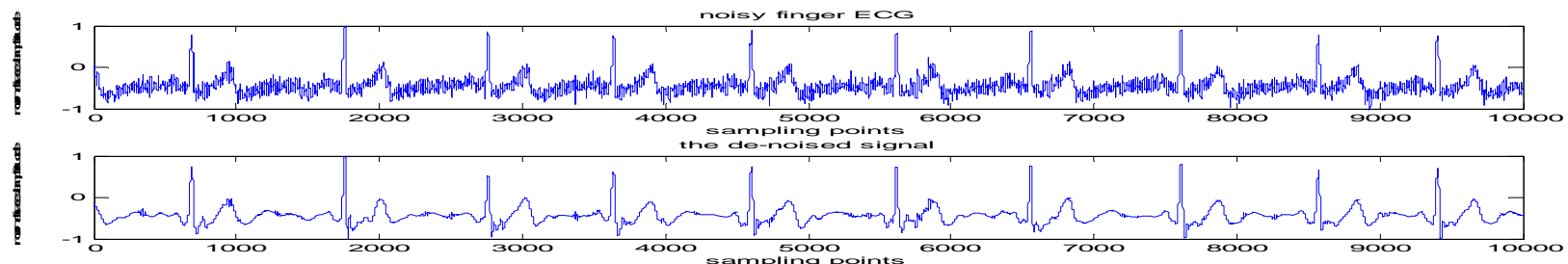

FIGURE VIII. COMPARISON BETWEEN BEFORE AND AFTER OF FINGER ECG DE-NOISING WHICH SIGNAL FROM CYBHI
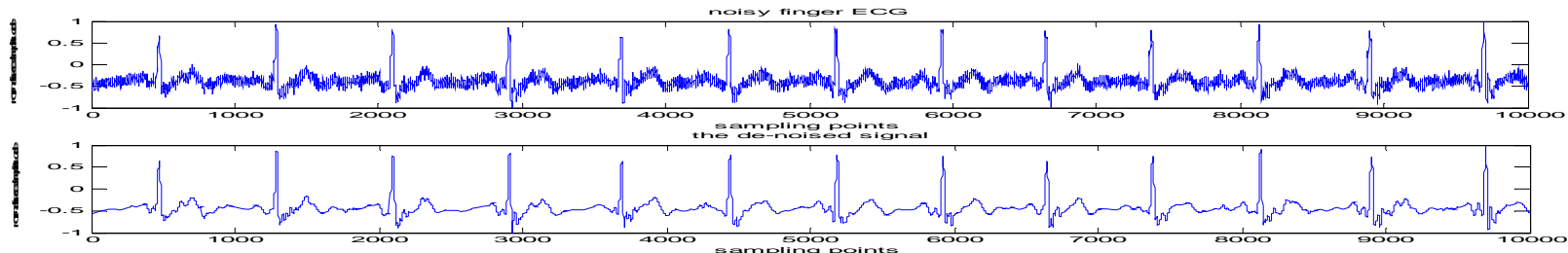

FIGURE IX. COMPARISON BETWEEN BEFORE AND AFTER OF FINGER ECG DE-NOISING WHICH SIGNAL FROM SURFACE ECG DATA
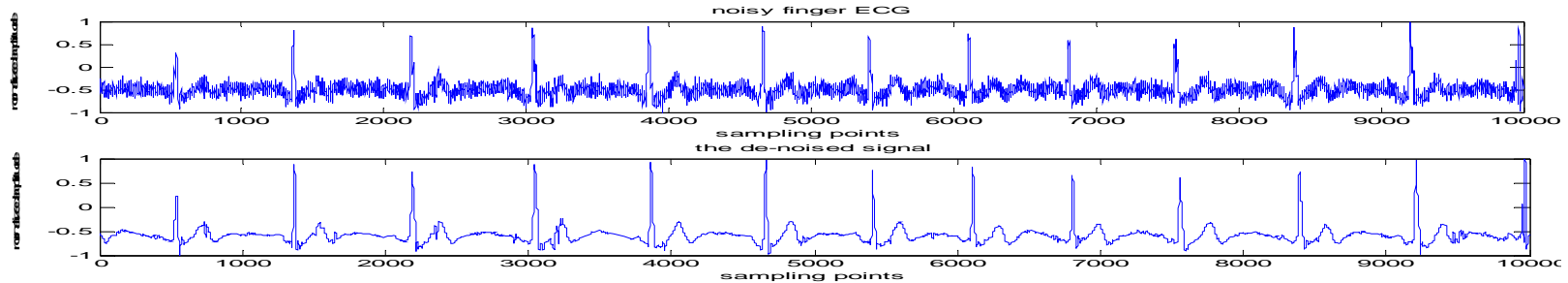

FIGURE X. COMPARISON BETWEEN BEFORE AND AFTER OF FINGER ECG DE-NOISING WHICH SIGNAL FROM FINGER ECG DATA 


\section{CONCLUSION}

In this paper, an optimal value is obtained by genetic algorithm to fit the wavelet threshold, and this value can dynamically changed in accordance with the characteristic of finger ECG. So it brings a least reconstruction error between the reconstructed signal and the original signal and achieve a better de-noising. The proposed GA based wavelet method of finger ECG de-noising is simulated by synthetic signal and finger signals from three finger ECG database. Results show that the proposed method get the better performance comparing to other algorithms, and better able to remove ECG signals collected under non constrained environment.

\section{ACKNOWLEDGMENT}

This research was supported by Zhejiang Provincial Natural Science Foundation of China under Grant No. LY14F010021 and by science technology department of Zhejiang Province commonweal project Grant No. 2015 C31086

\section{REFERENCES}

[1] Donoho D L, Johnstone J M. Ideal spatial adaptation by wavelet shrinkage[J]. Biometrika, 1994, 81(3): 425-455.

[2] Guangshu HU. Modern Signal Processing Guide.[M]. BeiJing: Tsinghua University press, 2004: 397-405.

[3] Donoho D L, Johnstone I M. Neo-Classical Minimax Problems, Thresholding and Adaptive Function Estimation[J]. Bernoulli, 1996, 2(1):39-62.

[4] Holland J H. Genetic algorithms and the optimal allocation of trials[J]. Siam Journal on Computing, 1973, 2(2):88-105.

[5] Singh B N, Tiwari A K. Optimal selection of wavelet basis function applied to ECG signal denoising[J]. Digital Signal Processing, 2006, 16(3): 275-287.

[6] Silva H P D, Lourenço A, Fred A. Check Your Biosignals Here: A new dataset for off-the-person ECG biometrics[J]. Computer Methods \& Programs in Biomedicine, 2014, 113(2):503-514.

[7] Chan A D C, Hamdy M M, Badre A, et al. Wavelet Distance Measure for Person Identification Using Electrocardiograms[J]. IEEE Transactions on Instrumentation \& Measurement, 2008, 57(2): 248-253.

[8] Saikia, B.J, Baruah, U. A GA-NN based wavelet method of speech signal denoising[C]// Computer Communication and Informatics (ICCCI), 2015 International Conference on. IEEE, 2015.

[9] Z. Zhao, M. Lv, X. Zhang. ECG De-noising Based on Translation Invariant Wavelet Transform and Overlapping Group Shrinkage[J]. Sensors \& Transducers (1726-5479), 2014, 177(8):54-62. 